\title{
Recherches féministes
}

\section{Contraintes socioculturelles et développement économique en Inde centrale : le cas des femmes saharia}

\section{Abha Chauhan}

Volume 8, numéro 1, 1995

Femmes, populations développement

URI : https://id.erudit.org/iderudit/057821ar

DOI : https://doi.org/10.7202/057821ar

Aller au sommaire du numéro

Éditeur(s)

Revue Recherches féministes

ISSN

0838-4479 (imprimé)

1705-9240 (numérique)

Découvrir la revue

Citer cet article

Chauhan, A. (1995). Contraintes socioculturelles et développement économique en Inde centrale : le cas des femmes saharia. Recherches féministes, 8(1),

99-109. https://doi.org/10.7202/057821ar
Résumé de l'article

Le présent article rend compte d'une recherche effectuée dans la communauté saharia en Inde centrale. Il montre que les programmes de développement économique ont des effets différents sur les hommes et les femmes, compte tenu des contraintes socioculturelles qui s'exercent sur ces dernières. L'auteure insiste sur la nécessité d'étudier les femmes des groupes «tribaux» de l'Inde à la lumière de leur propre culture et en tenant compte de la position qu'elles occupent dans ces communautés. 


\section{Contraintes socioculturelles et développement économique en Inde centrale : le cas des femmes saharia*}

\section{Abha Chauhan}

Le présent article aborde un certain nombre de questions à caractère méthodologique concernant la condition des femmes des groupes tribaux ${ }^{1}$ dans le contexte historique et culturel indien, où les études sur le changement social sous-estiment l'importance des femmes de ces groupes. II présente une analyse de la situation des femmes chez les Saharia, une tribu pauvre et peu nombreuse de l'Inde centrale. L'accent est mis sur les contraintes culturelles ${ }^{2}$ que subissent ces femmes dans le processus de développement économique, car une meilleure compréhension de ces contraintes est nécessaire au succès des programmes de développement conçus à leur intention. Le rôle actuel des femmes dans les communautés indigènes et leurs réactions aux nouveaux changements sont aussi mis en rapport avec la position de celles-ci dans la culture traditionnelle et les relations sociales et politiques qui la caractérisent.

Selon le recensement de 1991, 70 millions de personnes appartiennent à l'une ou l'autre des 400 tribus qui existent en Inde, soit 8,08 p. 100 de la population. Les Saharia constituent l'une des Scheduled Tribes de I'Inde et font aussi partie des 73 Groupes tribaux primitifs (Primitive Tribal Groups) (PTG), définis selon trois critères : un niveau technologique pré-agricole; un faible taux d'alphabétisation; une population stagnante ou en baisse. Les Saharia vivent principalement dans l'Etat du Madhya Pradesh (dans les districts de Morena, Gwalior, Shivpuri et Guna), en Inde centrale; au recensement de $1981^{3}$, le groupe comptait 261821 personnes. Sous la confédération marhatha, cette région faisait partie de l'État princier de Gwalior, qui demeura en grande partie entre les mains de la Central India Agency sous I'administration britannique. Avant cela, le Gwalior a connu plusieurs souverains rajput et moghol. Des spécialistes pensent que la population saharia, auparavant maîtresse de cette région, aurait été repoussée vers les montagnes et les forêts de l'intérieur et dépossédée de ses terres fertiles (Saxena 1964 : 25-30). On classe les Saharia dans le groupe

* Traduit de l'anglais par Catherine Zekri et Huguette Dagenais.

1. Les termes tribes, tribal groups, tribal women, tribal men employés dans la version orignale en anglais font référence à des catégories administratives de l'État indien, elles-mêmes héritées de la colonisation britannique. C'est la raison pour laquelle, dans la traduction française, nous avons conservé ces termes, même s'ils ne sont plus en usage aujourd'hui en sciences sociales, où l'on parle plutôt de communautés ou de groupes " autochtones" .

2. L'expression « contraintes culturelles " comprend ici à la fois les aspects propres à la culture tribale et ceux qui sont acquis du fait d'interactions avec d'autres cultures, qui font obstacle actuellement au développement économique des communautés indigènes.

3. Les États voisins du Rajasthan et de I'Uttar Pradesh comprennent aussi une population saharia. 
tribal munda ou kolar. Leur nom figure dans les anciens contes épiques religieux et dans la littérature védique.

Les contacts entre la culture indigène saharia et les cultures extérieures ont eu pour résultat l'intégration des Saharia dans la hiérarchie sociale régionale ${ }^{4}$, à un échelon équivalent à celui des harijan ou intouchables. Les Saharia ont ainsi adopté des attitudes et des perceptions de la réalité qui font maintenant partie de leur culture. C'est pourquoi une connaissance de la culture des Saharia est essentielle pour comprendre leurs réactions par rapport au changement social actuel et, surtout, comme on le verra, les effets de celui-ci.

Toutefois, le changement que connaissent les groupes tribaux touche différemment les hommes et les femmes et, par conséquent, leurs réactions au changement. On peut comprendre cette différence en analysant d'abord la position des femmes à l'intérieur de la culture saharia, puis, dans une perspective historique, dans les rapports de celle-ci avec les cultures extérieures. C'est ce que je tenterai de faire dans la première partie de mon article, alors que, dans la deuxième partie, j'analyserai plus précisément le processus de développement et ses conséquences sur les femmes des groupes tribaux.

Les données présentées ici proviennent principalement d'une recherche de terrain effectuée, de 1990 à 1992, dans quatre villages du district de Shivpuri. Ces villages sont Jingni, dont la population est de 500 personnes (à 100 p. 100 tribale), Mehlauni qui en compte 600 (dont 80 p. 100 appartiennent à des groupes tribaux), Baglon où l'on en trouve 300 (60 p. 100) et Digdoli qui en abrite 800 (35 p. 100). Selon le recensement de 1981, la population du district était de 865930 personnes, parmi lesquelles 86471 (10 p. 100) appartenaient à des groupes tribaux, dont 75471 au groupe saharia. La population tribale étant peu nombreuse dans les villages, les informations furent recueillies à la fois par sondage, par observation participante ou non et par entrevues, comportant des questions fermées et des questions ouvertes. J'ai aussi tenu un journal de bord. En général, je me postais dans un endroit du village où la population se rassemblait spontanément. Bien que les informations qui suivent s'appuient très largement sur des entretiens non structurées ou fortuits que j'ai eus avec les Saharia de cette manière et en participant aussi bien à leur vie quotidienne qu'à leurs activités spéciales, 100 personnes (50 âgées de plus de 50 ans et 50 , moins âgées) furent interviewées plus en profondeur. La communication n'a posé aucun problème, car elle s'est déroulée en braj-bhasa, un dialecte de l'hindi, langue maternelle de l'auteure de la présente étude.

\section{L'évolution de la situation des femmes saharia}

\section{La position des femmes dans la culture saharla traditionnelle}

Dans la culture traditionnelle des Saharia, les différents aspects de la vie sociale tournaient autour du fonctionnement du panchayat, l'institution assurant

4. II existe plus de 2500 castes endogames en Inde. Dans la région où a été réalisée notre recherche, la hiérarchie sociale est la suivante. Les brahmanes (prêtres) constituent la caste supérieure; viennent ensuite les rajput (rois, dirigeants), les bania (marchands, commerçants), puis les classes inférieures, telles que les kirar et les ahir (bergers et paysans), les harijan (considérés comme intouchables) et les saharia (groupe tribal). 
le contrôle et l'ordre social dans la communauté. Le panchayat est composé des anciens du village dirigés par un chef, le patel. Les femmes ne peuvent être membres du panchayat, ni participer à ses réunions. Elles n'ont donc pas voix au chapitre en ce qui concerne les questions sociales importantes et elles n'exercent aucun pouvoir. Le panchayat intervient, en effet, sur tous les plans (religieux, social et économique), sauf sur le plan familial. II ne prend jamais position dans les conflits familiaux, tels que l'alcoolisme, la violence des hommes contre les femmes et la négligence à l'égard des enfants, ou lorsque l'homme ne subvient pas aux besoins de sa famille. Les peines imposées par le panchayat sont ordinairement des amendes ou des châtiments corporels. Ces derniers peuvent être assez sévères pour les femmes, qui sont facilement taxées de churel (mauvais esprit) ou panchdevli ${ }^{5}$, selon la décision des membres du panchayat, en collaboration avec le marhi (chef religieux) et l'ojha (sorcier). De plus, comme les rituels sont centrés sur le cycle menstruel et la naissance, les femmes doivent se soumettre à divers tabous et restrictions.

Dans l'économie tribale traditionnelle, les femmes avaient un rôle important à jouer. La forêt abondait dans cette région et les communautés indigènes jouissaient d'une autonomie complète quant à son exploitation et à ses ressources. Les principaux modes de production étaient la culture sur brûlis ainsi que la cueillette et la transformation des produits de la forêt. La chasse, la pêche, la fabrication de cordes et de paniers procuraient un revenu accessoire. Les 50 personnes âgées de 50 ans ou plus ( 24 hommes et 26 femmes) que j'ai interviewées ont parlé longuement de leur participation à la culture sur brûlis et à ses activités connexes, de même que de la vie en régime princier. Dans la culture sur brûlis, appelée sur-dahiya ou, plus communément, soor et pratiquée dans cette région jusqu'au début des années 1940, les hommes accomplissaient principalement la coupe et l'abattage des arbres, puis le brûlage; les femmes s'occupaient de la préparation, de l'ensemencement et du nivellement des sols, du désherbage, puis de la récolte, du stockage et de la vente. La collecte des produits de la forêt était surtout une activité féminine. Ces produits servaient à l'alimentation, à des usages médicinaux et, par la vente ou le troc, à l'acquisition d'autres nécessités comme le sucre, l'huile, les épices, les céréales ou les vêtements. La transformation des produits forestiers, comme la fabrication de charbon de bois, de boisson à la fleur de mahua, d'huile de gillu (servant de combustible) ou de savon, incombait autant aux hommes qu'aux femmes. Outre la cuisine de tous les jours, les femmes préparaient à partir de ces produits une variété de plats nourrissants. Comme elles s'occupaient aussi quotidiennement des enfants, de la nourriture et de l'approvisionnement en bois, en fourrage et en eau, leur charge de travail était plus lourde que celle des hommes. Toutefois, les autres membres du clan et du village apportaient un peu d'aide.

Les femmes saharia jouissaient d'une grande liberté en ce qui concerne les questions sociales telles que le mariage, le divorce ou le remariage. Le mariage des enfants n'existait pas; on offrait la dot (bride-wealth) et les cérémonies du mariage étaient simples ${ }^{6}$. Même si la résidence du couple marié était virilocale et la

5. Une femme qualifiée de panchdevli n'a plus aucun statut et devient la propriété des hommes du panchayat (panches), qui peuvent l'utiliser à leur guise.

6. Dans le village de Jingni, j'ai pu assister au mariage d'une jeune femme et constater qu'il s'agissait d'une cérémonie très simple, comparativement au mariage des 
famille, nucléaire, les femmes ne rencontraient guère d'obstacles dans leur adaptation à cette nouvelle vie, puisqu'on construisait une nouvelle maison ou tapara pour le couple, et la femme était responsable seulement de son propre travail et de celui de son mari, le reste des tâches étant accomplies avec les autres membres de la communauté ${ }^{7}$.

Si les femmes jouaient un rôle indispensable dans l'économie indigène et jouissaient d'une liberté en ce qui a trait à certaines questions sociales, les hommes, quant à eux, détenaient un pouvoir considérable du fait qu'ils prenaient les décisions importantes au sein du panchayat. Ils occupaient les positions clés de chefs politiques et culturels, et ce sont eux qui construisaient l'idéologie. On jugeait les femmes incapables d'accomplir les activités de gestion du panchayat. On les considérait comme des commères, incapables de tenir des propos sérieux ou de garder une information secrète; leur principale sphère d'activité était et devait demeurer la maison, les enfants, les champs et la forêt. La culture saharia traditionnelle présente donc un paradoxe entre, d'une part, la participation active des femmes à la vie économique et sociale et, d'autre part, leur subordination sur les plans politique et culturel.

\section{L'ordre princler et le changement dans le statut des femmes saharia}

Au cours du $X X^{\theta}$ et au début du $X X^{\theta}$ siècle, d'importants changements ont eu lieu sous le règne des Maratha au Gwalior, alors que furent instaurés les systèmes des jagirs (petites propriétés allouées en guise de rétribution aux fonctionnaires et aux guerriers) et des zamindari ${ }^{8}$, en même temps qu'on mettait sur pied le Département des forêts dans le but de protéger les ressources forestières. Ces événements contribuèrent à l'immigration de fonctionnaires, d'entrepreneurs, d'usuriers et de commerçants. Les Saharia devaient dorénavant payer des impôts aux autorités pour pratiquer l'agriculture sur brûlis alors que les forêts étaient attribuées, sur une base annuelle, à des entrepreneurs, qui les exploitaient en utilisant une main-d'œuvre saharia. Les Saharia devaient encore accomplir des corvées (begar) pour l'État et les zamindari. De plus, les commerçants et les usuriers en faisaient des halis (travailleuses et travailleurs liés par les dettes). Plusieurs enquêtes montrent qu'il y avait de la discrimination dans la rémunération des Saharia pour leurs services et pour les produits forestiers collectés (Shastri 1991: 68-69). Des vieilles personnes de la communauté saharia ont mentionné qu'elles avaient extrêmement peur des fonctionnaires et se cachaient souvent à leur approche.

La communauté saharia a été privée de beaucoup d'avantages socioéconomiques et placée au palier inférieur de la hiérarchie régionale. En

femmes non indigènes. Aucun prêtre brahmane n'était présent; la mariée reçut quelques ustensiles et des vêtements, et il n'y eut pour toute nourriture que du pain (chapatis) et une portion de curry dilué.

7. Une tapara (maison à une chambre) est construite pour chaque couple nouvellement marié. Cette coutume est différente de celles des castes hindoues supérieures des villages, chez lesquelles les familles vivent ensemble et où la nouvelle belle-fille subit la réclusion et de nombreuses restrictions; elle a aussi une lourde charge de travail.

8. Dans ce système, le zamindar, un intermédiaire, paie aux dirigeants de l'État les taxes perçues des fermiers. 
conséquence, la population a développé un sentiment de peur et d'infériorité qui, d'une part, lui a fait éviter le contact avec les non-indigènes et, d'autre part, a fait cesser certaines habitudes, telle la consommation de céréales et de carrion (viande non cuite) (Census Report 1901 : 132). Parallèlement, les Saharia ont tenté d'imiter les activités sociales et culturelles des castes supérieures, dont certaines se rapportant aux femmes et à leur position dans la société tribale ${ }^{9}$. Les activités des femmes saharia n'étaient guère prestigieuses comparées à celles des femmes des hautes castes et leur statut était établi en conséquence. Les coutumes saharia associées au divorce et au remariage, la liberté des femmes dans leurs contacts avec les hommes et leur travail aux champs étaient méprisés. Plusieurs pensent que les femmes saharia seraient devenues des proies faciles pour les hommes influents, ce qui aurait obligé les hommes saharia à les garder autant que possible à la maison. C'est ainsi que des valeurs et les coutumes patriarcales, comme le purdah, la monogamie, le mariage des enfants, le paiement de la dot par la famille de la mariée à celle du mari (dowry), furent introduites dans la vie des Saharia au contact de la culture non tribale et que s'ajoutèrent de nouvelles contraintes à celles que subissaient déjà les femmes saharia du fait de leur rôle politique négligeable et de la discrimination socioreligieuse à leur égard. Toutes ces contraintes sont encore renforcées par le processus de développement économique, amorcé dans cette région après l'Indépendance.

\section{L'ordre national démocratique et les groupes tribaux}

Avec l'accession à l'Indépendance, proclamée en 1947, et l'adoption de la Constitution, en 1950, I'Inde instaurait un ordre politique socialiste, séculier et démocratique, dans lequel l'État s'engageait à ne faire aucune discrimination sur la base de la religion, de la race, de la caste, du sexe ou du lieu de naissance (article 15). Selon la Constitution indienne, l'État a même la responsabilité de promouvoir, avec un soin particulier, les intérêts des Scheduled Tribes en matière d'éducation et d'économie, en plus de devoir protéger ces communautés contre l'injustice et toute forme d'exploitation (article 16.4). II existe des subventions spéciales du gouvernement central en vue d'aider les états à implanter des programmes de développement (article 275.1) et, depuis la deuxième moitié des années 1970, des sous-plans sont mis en oeuvre pour favoriser le développement intégré des communautés tribales.

Cela suggère qu'un changement culturel et structurel considérable s'est produit par le passage d'un régime princier à un régime démocratique et d'un système de castes, qui institutionnalisait les inégalités, à un système égalitaire. Un changement d'un nouveau type est donc en cours. Cependant, il y a un écart évident entre les lois et leur application. Cela est particulièrement vrai pour les Saharia, qui forment une minorité parmi les communautés indigènes et qui sont

9. M.N. Srinivas $(1966: 6)$ désigne sous le nom de «sanscritisation» le processus de mobilité sociale qui se produit graduellement dans la société indienne et «par lequel une caste hindoue inférieure modifie ses habitudes, ses rituels, son idéologie et son mode de vie pour ressembler davantage à une caste supérieure, souvent celle des « deux fois nés [brahmanes] ". Parmi les traits culturels ainsi adoptés par les Saharia se trouvent l'abandon de toute nourriture non végétarienne et de l'alcool et l'imposition aux femmes de contraintes rigides. 
depuis longtemps en contact avec d'autres groupes. On a noté d'ailleurs que dans les communautés où l'influence du système de castes a été très profonde, comme c'est le cas chez les Saharia, le changement se produit lentement (Chauhan 1990).

Depuis l'Indépendance, il y a deux domaines où le changement a touché de manière significative la vie des communautés indigènes. Le premier domaine est la forêt, le second, la terre. Pendant des siècles, la forêt a fourni aux Saharia I'habitat, les moyens de subsistance, un lieu de culte, bref elle a été au centre de leur vie sociale et culturelle. L'Indian Forest Act a été adopté en 1927 et la politique forestière nationale (National Forest Policy) a été établie en 1952. Par ces mesures, on a interdit formellement la culture sur brûlis et la chasse, les revendications des communautés tribales ont été assujetties à l'intérêt national et leurs divers droits et privilèges sont devenus de simples concessions. Un grand nombre de lois et de règlements formels virent ainsi le jour, et les personnes illettrées des communautés indigènes se trouvèrent placées à la merci d'entrepreneurs, d'intermédiaires et de fonctionnaires forestiers aux pouvoirs illimités. Dans la région habitée par les Saharia, il est aujourd'hui défendu de prendre des produits forestiers d'importance, tel le bois d'œuvre. En outre, un grand nombre de produits forestiers d'importance secondaire ont été nationalisés, plaçant les Saharia sous la dépendance des entrepreneurs. Car il est permis aux Saharia de vendre aux intermédiaires certains produits secondaires, mais leur vente rapporte peu d'argent. Une enquête menée dans les quatre villages mentionnés précédemment révèle que 90 p. 100 des personnes employées à la collecte des produits secondaires sont des femmes, ce qui démontre que cette activité génère des revenus, bien que ceux-ci soient maigres. Ces femmes affirment toutefois qu'elles doivent passer plus de temps que par le passé à la recherche de ces produits, car ils se font considérablement plus rares au fil des années.

La signification de la terre dans la vie des communautés indigènes doit aussi être considérée dans le contexte des réformes foncières introduites depuis l'Indépendance. Les réformes foncières comprenaient la réforme de la tenure foncière, la consolidation des propriétés fragmentées, l'imposition d'un plafond sur les prix, l'abolition des intermédiaires et la mise à jour régulière des registres, outre la fusion de plusieurs États princiers, dont le Gwalior, en une seule unité administrative et politique. Les réformes avaient pour buts la redistribution des terres aux classes les moins favorisées et l'amélioration des conditions faites à ceux et celles qui les travaillent, en vue de faire cesser l'exploitation et d'assurer la sécurité foncière. La distribution des terres disponibles aux pauvres et aux personnes qui n'en possédaient pas, dont une grande partie appartenait aux groupes tribaux, est présentement en cours dans différents États. L'allocation de terres aux Saharia et l'assistance technique pour rendre ces terres cultivables sont en cours également. En outre, des prêts sont accordés pour encourager le travail à son propre compte et les projets générateurs de revenus, dans le cadre de divers programmes de développement rural intégré de même que dans les sous-plans à l'intention des groupes tribaux. Examinons maintenant l'impact de ces programmes de développement sur les femmes saharia. 


\section{Les programmes de développement économique et la marginalisation des femmes saharia}

Le passage d'une agriculture itinérante à une agriculture sédentaire a profondément transformé le mode de vie des Saharia. Ce fut un processus lent mais soutenu, amorcé dans les années 1920, lorsque le groupe abandonna la culture soor. Depuis le début des années 1980, le processus s'est accéléré avec l'allocation de terres à mettre en culture. II y a deux raisons à l'abandon de l'agriculture sur brûlis : d'une part, la limitation des droits d'exploitation et la diminution des ressources forestières; d'autre part, la possibilité pour les Saharia de travailler sur les terres de paysans riches, terres acquises des administrations d'autrefois. Depuis le début des années 1970, les Saharia bénéficient de plusieurs éléments du confort moderne et de services, tels que l'électricité, l'eau courante, un réseau routier, des écoles, des banques et des bureaux de poste.

Toutefois, en tant que cultivatrices, les femmes saharia sont dorénavant privées de leur terre et de leurs droits d'usage foncier et, en tant que cueilleuses, elles n'ont plus accès aux ressources forestières secondaires, ce qui les rend plus dépendantes que par le passé. Un grand nombre d'entre elles travaillent comme ouvrières agricoles sur les terres de riches propriétaires. Elles sont généralement engagées pour la récolte des arachides, des tubercules ou la coupe des plants de moutarde, de blé ou de canne à sucre. L'impact de ces changements est visible dans la structure occupationnelle des communautés et dans la division sexuelle des tâches. De plus, la charge de travail des femmes a doublé parce que, outre leurs activités de production et de reproduction, elles continuent d'assumer la responsabilité de l'approvisionnement en combustible, en fourrage et en eau, sans parler de l'insécurité accrue dans laquelle elles vivent à cause du peu de possibilités d'emploi pour les femmes. II n'est donc pas étonnant que les femmes saharia préfèrent l'époque de la culture sur brûlis, car il y avait du travail et de la nourriture tout au long de l'année.

Les activités des hommes, quant à elles, se diversifient, puisque ceux-ci peuvent travailler comme cultivateurs, ouvriers de la construction, vendeurs d'artisanat et de produits forestiers, ou comme employés dans les programmes de foresterie communautaire. À l'époque de l'enquête, pas une seule femme des quatre villages étudiés ne travaillait en foresterie, dans la construction de routes ou le creusage de puits, par exemple. Très peu d'hommes d'ailleurs ont accès à ce genre d'emplois : en moyenne, quatre ou cinq seulement par village, et pour trois mois par année seulement. Le réseau de soutien aux familles, que formaient les hommes des villages et des clans, s'est réduit considérablement à mesure qu'un nombre grandissant d'entre eux ont développé une dépendance à l'alcool local. Subvenir aux besoins de la famille et s'occuper des enfants relèvent donc de plus en plus des femmes, ce qui explique la division des tâches, plus prononcée que par le passé. Cette tendance augmente encore la marginalisation des femmes dans l'économie tribale et leur " domestication ". Malgré leur double tâche, en effet, les femmes des communautés indigènes sont de plus en plus perçues comme "inactives " ${ }^{10}$ ou comme une main-d'œuvre

10. La sous-estimation du rôle économique des femmes dans la société est due en grande partie au concept occidental de « travail » qui désigne la participation à la production marchande ou au travail salarié. Voir Beneria (1982). 
" marginale ", alors que les hommes sont toujours considérés comme la maind'œuvre "principale". Ainsi, dans le recensement de 1981, parmi les 86471 membres des groupes tribaux du district de Shivpuri, 33742 étaient sur le marché du travail, soit 24353 hommes et 9389 femmes (The District Census Handbook 1981). Ces différences sont encore plus frappantes lorsqu'on examine les programmes de développement à l'intention des groupes tribaux et leurs effets sur les femmes.

Ainsi, depuis plus d'une décennie, dans cette région, les terres sont distribuées aux hommes des communautés tribales, pour être ensuite transmises de père en fils. La population de ces communautés étant illettrée et ne connaissant souvent rien aux nouvelles lois en matière d'agriculture sédentaire, elle éprouve une certaine confusion devant ce changement. Toutefois, celui-ci comporte deux aspects qui affectent les femmes de façon particulière. D'une part, on assiste à l'introduction du concept de propriété privée dans une société caractérisée par la propriété collective des terres forestières. D'autre part, dans les programmes de développement, les terres sont attribuées uniquement aux hommes chefs de ménage, sans tenir compte de l'usage et du contrôle qu'en avaient traditionnellement les femmes indigènes. De plus, dans le cas des hommes qui maltraitent, battent ou abandonnent leur femme, celle-ci se retrouve sans recours : elle n'a plus ni maison, ni propriété, ni terre. Car, bien qu'on attende des hommes qu'ils partagent leurs avoirs, rien ne les y oblige, ce qui revient, dans les faits, à désavantager grandement les femmes.

La situation de certaines familles saharia s'est cependant améliorée. Grâce aux technologies nouvelles, les familles ont commencé à varier leurs cultures. Mais cela a engendré des inégalités de classe : certaines personnes ont davantage de terre, d'autres en ont moins et d'autres, pas du tout. Les femmes des familles aisées cessent de travailler aux champs, se confinant aux activités domestiques. L'inégalité est visible aussi dans l'enseignement des techniques d'irrigation et des nouvelles techniques en général, qui est également dispensé seulement aux hommes, alors que dans l'économie traditionnelle les femmes maîtrisaient bien le maniement des outils. En 1991, par exemple, dans le village de Mehlauni, on a fourni une pompe diesel à deux hommes et une pompe électrique à un autre pour l'irrigation, mais eux seuls pouvaient les faire fonctionner, pas les femmes de leur famille. Par contre, aucune technique susceptible de réduire la charge de travail des femmes n'a été introduite. Ainsi, au fur et à mesure de la marginalisation des femmes, de leur domestication et de leur plus grande dépendance à l'égard des hommes, les inégalités de classe renforcent les inégalités de sexe.

De plus, le fait que toutes ces mesures soient écrites et formalisées dans des règlements intensifie les problèmes des personnes illettrées. Cela engendre d'autres types de contraintes pour les femmes qui manquent généralement de formation et d'instruction et qui, de ce fait, ont du mal à entreprendre de nouvelles activités économiques. Ces contraintes sont évidentes, notamment dans la procédure entourant l'octroi de prêts aux femmes. Dans le cadre des divers programmes de développement à l'intention des groupes tribaux, les femmes saharia reçoivent, en effet, des prêts subventionnés à $50 \mathrm{p}$. 100 pour mettre sur pied leur propre entreprise et ainsi gagner leur vie de façon autonome. Dans les quatre villages à l'étude, de 1987 à 1992, 50 personnes, dont 21 femmes, ont reçu de tels prêts. Cependant, pour diverses raisons, ces femmes 
n'ont pu utiliser cet argent, ni créer de nouvelle activité économique. Par exemple, les prêts furent alloués sans que les femmes bénéficiaires aient reçu de formation adéquate; la plupart d'entre elles ne savaient pas comment gérer les moyens mis à leur disposition parce que ceux-ci ne concernaient pas l'économie forestière. De plus, les femmes des groupes tribaux sont spécialisées dans la récolte et dans la transformation des produits de la forêt, mais aucune entreprise de ce type ne pouvait être mise sur pied. En fait, les prêts étaient accordés pour la fabrication de confitures et de marinades, des produits traditionnellement préparés par les femmes dans leur foyer. Le choix de telles activités témoigne de l'ignorance des personnes qui conçoivent les programmes quant au rôle économique des femmes des groupes tribaux et reflète aussi leur propre conception du travail de ces femmes. Par ailleurs, comme ces programmes sont instaurés par des hommes au sein des panchayats, les femmes sont réticentes à y participer. Toutes celles qui participent le font sur l'insistance d'hommes, euxmêmes influencés par d'autres hommes, extérieurs aux communautés. II arrive fréquemment que les femmes reçoivent moins d'argent que prévu, comme il arrive aussi que les prêts soient effacés. Dans ce dernier cas, le fait de pouvoir utiliser l'argent comme bon leur semble, sans avoir à le rembourser, a provoqué paresse et corruption chez certaines Saharia, auparavant réputées pour leur ardeur au travail et leur honnêteté. Quoi qu'il en soit, les femmes considèrent cet argent comme un revenu d'appoint et n'en sont pas dépendantes, car les montants accordés, en plusieurs versements, sont faibles et ne satisfont pas à leurs besoins élémentaires, sans parler du temps et des formalités nécessaires pour les obtenir.

Enfin, les programmes de développement sont imposés aux femmes des communautés tribales sans qu'on leur fournisse la formation et les infrastructures nécessaires. En 1981, le taux d'alphabétisme dans l'État du Madhya Pradesh était de 27,87 p. 100 pour l'ensemble de la population totale, de 10,68 p. 100 pour les groupes tribaux et de 3,60 p. 100 pour les femmes de ces groupes. Sur les 261822 Saharia, 7180 personnes étaient alphabétisées, dont 495 femmes seulement (Census of India 1981). Poursuivre des études ne signifie pas seulement l'assurance de gagner plus d'argent plus tard, mais aussi, dans l'immédiat, un investissement de temps et une diminution de revenus. Or, les Saharia constituent une tribu pauvre et exploitée depuis bien des années, dont les membres ont peu de possibilités d'emploi, en raison de l'intrusion d'autres groupes sur leur territoire et des restrictions imposées à leur mode de vie, centré sur la forêt. De plus, les femmes saharia ne tirent pas vraiment avantage de l'éducation; le taux d'inscription à l'école est beaucoup moins élevé chez les filles que chez les garçons et le taux d'abandon, lui, est plus élevé. Par ailleurs, comme la communauté préfère se déplacer et migrer pendant la saison de la récolte ${ }^{11}$, il devient difficile de lui fournir l'enseignement de manière systématique et continue. La division sexuelle du travail, devenue plus rigide chez les Saharia sous l'influence des valeurs patriarcales dominantes dans la région, est également responsable de l'écart entre les sexes en matière d'éducation. De

11. Selon Fuchs (1992: 866), bon nombre de groupes tribaux ont conservé leur mode de vie nomade et communautaire pour défier cette nouvelle situation. L'attrait de la vie nomade serait très fort également chez les Saharia, comme l'a noté Bairathi (1991: 235). 
même, l'idéologie selon laquelle la place des femmes est à la maison et leur travail devrait se réduire aux tâches domestiques, associée au maintien de coutumes comme le mariage des enfants, empêche les membres des tribus d'accorder de l'importance à l'éducation des femmes.

\section{Conclusion}

En somme, les programmes de développement économique à l'intention des tribus en Inde ont considérablement affecté les femmes saharia. Les femmes des communautés tribales sont dorénavant privées de leurs droits d'usage de la terre et des produits de la forêt. Leur lutte quotidienne pour se procurer les produits de base (nourriture, eau et combustible) s'est accentuée. Les nouvelles activités de production ne leur sont guère ouvertes, faute des connaissances et de la formation appropriées. Même les prêts censés les aider à acquérir l'autonomie financière portent la marque d'une conception patriarcale du travail féminin et d'une méconnaissance des activités traditionnelles des groupes en question. De plus, l'écart augmente aussi bien entre les classes sociales qu'entre les hommes et les femmes.

Le cas des femmes saharia montre donc que connaître la position des femmes au sein de leur culture, le rôle clé qu'elles jouent dans l'économie et dans la division sexuelle du travail ainsi que le double fardeau qu'elles doivent assumer du fait de leurs activités de production et de reproduction est absolument nécessaire à l'élaboration des programmes de développement économique. Le présent article aura été utile s'il réussit à montrer aussi que l'emprunt ou l'imposition de traits culturels entraîne des contraintes pour les populations visées et qu'il est essentiel, dans les programmes de développement économique destinés aux femmes des groupes tribaux en Inde, de comprendre l'ensemble de leur culture.

\section{Abha Chauhan \\ Baikunthi Devi College \\ Agra, Inde}

\section{RÉFÉRENCES}

BAIRATHI, Shashi

1991 Tribal Culture, Economy and Health. Jaipur, Rawat Publications.

BENERIA, Lourdes (dir.)

1982 Women and Development. New York, Praeger.

CENSUS OF INDIA

1981 Special Tables on Scheduled Tribes. Series II, M.P., Part IX (VIII) : Table 6A and B and 7.

CENSUS REPORT

1901 Gwalior State. Vol. XXI, Part 1.

CHAUHAN, Abha

1990 Tribal Women and Social Change in India. Etawah, A.C. Brothers. 
FUCHS, Steven

1992 « Indian Tribals and the Forest ",. in Buddhadeb Chaudhari (dir.), Economy and Agrarian Issues. New Delhi, Inter India Publications.

SAXENA, R.P.

1964 Tribal Economy in Central India. Calcutta, Mukhopadhyaya.

SRINIVAS, M.N.

1966 Social Change in Modern India. Los Angeles, California.

THE DISTRICT CENSUS HANDBOOK, SHIVPURI DISTRICT

1981 Census of India. Series II, M.P., Part VII-B. 